\title{
UPPER BOUNDS FOR THE ESSENTIAL DIMENSION OF $E_{7}$
}

\author{
MARK L. MACDONALD
}

\begin{abstract}
This paper gives a new upper bound for the essential dimension and the essential 2-dimension of the split simply connected group of type $E_{7}$ over a field of characteristic not 2 or 3 . In particular, $\operatorname{ed}\left(E_{7}\right) \leq 29$, and $\operatorname{ed}\left(E_{7} ; 2\right) \leq 27$.
\end{abstract}

The essential dimension of an algebraic group $G$ is a numerical invariant which measures the complexity of its $G$-torsors. The essential dimensions of the exceptional algebraic groups remain largely open (see [Re10] for a recent survey). For example, even if the base field is the complex numbers we only know that $9 \leq \operatorname{ed}\left(E_{8}\right) \leq 231$ ([CS06], [Le04]).

We will say that a $G$-variety $X$ is generically free if there is an open subset $U \subset X(\bar{k})$ on which $G(\bar{k})$ acts with trivial stabilizers, where $\bar{k}$ is an algebraic closure of the base field $k$. We will define the essential dimension of $G$ to be the minimum value of $\operatorname{dim}(X)-\operatorname{dim}(G)$, where $X$ is a generically free $G$-variety and there exists a $G$ equivariant dominant map $V \rightarrow X$ (also called a compression), such that $V$ is a generically free linear representation of $G$.

We assume throughout this paper that the characteristic of the base field $k$ is not 2 . Let $E_{n}$ denote the split simply connected group of that type. We have the lower bound $7 \leq \operatorname{ed}\left(E_{7}\right)$ from [CS06] (for characteristic zero, see [RY00]), and over the complex numbers, we know that three copies of $E_{7}$ 's faithful 56 dimensional representation $V_{56}$ is generically free [Po86, 13], and so is $V_{56} \times \mathbb{P}\left(V_{56}\right) \times \mathbb{P}\left(V_{56}\right)$. Therefore ed $\left(E_{7}\right) \leq$ $(56+55+55)-133=33$, which is the best known upper bound. In this paper we will improve this bound, and only assume the base field has characteristic not 2 or 3 .

For a prime $p$, we are also interested in the "localized" notion of essential $p$-dimension, which is often easier to compute. For primes $p$ not equal to 2 or 3 , we know ed $\left(E_{7} ; p\right)=$ 0 . Also, ed $\left(E_{7} ; 3\right)=3$ ([GR09] or [Ga09]). For $p=2$ we have $7 \leq \operatorname{ed}\left(E_{7} ; 2\right) \leq 33$ ([RY00], [CS06], [Mac11]). In this paper we improve the upper bound.

We will take the subgroup $E_{6} \rtimes \mu_{4} \subset E_{7}$ as defined in [Ga01, 3.5]. For every field extension $l / k$, inclusion induces a surjection $H^{1}\left(l, E_{6} \rtimes \mu_{4}\right) \rightarrow H^{1}\left(l, E_{7}\right)$ (see also [Ga09, $12.13])$; in other words, we can reduce the structure of $E_{7}$-torsors. In particular, this implies

$$
\operatorname{ed}\left(E_{7}\right) \leq \operatorname{ed}\left(E_{6} \rtimes \mu_{4}\right) \quad \text { and } \quad \operatorname{ed}\left(E_{7} ; 2\right) \leq \operatorname{ed}\left(E_{6} \rtimes \mu_{4} ; 2\right) .
$$

The new upper bounds will be achieved by using these inequalities. As always, we have $\operatorname{ed}\left(E_{7} ; 2\right) \leq \operatorname{ed}\left(E_{7}\right)$, but we do not know if this inequality is strict.

Consider $E_{7}$ 's fundamental faithful representation of dimension 56 (also known as its minuscule representation). As an $E_{6}$ representation it decomposes as $k \oplus k \oplus V \oplus V^{*}$, where $V$ and $V^{*}$ are $E_{6}$ 's two fundamental representations of dimension 27 (also known as its minuscule representations), and $k$ denotes a one-dimensional trivial representation. Let $w \in \mu_{4}$ be defined as in [Ga01,3.5], sending an element $\left(\alpha_{1}, \alpha_{2}, v_{1}, v_{2}\right)$ to $\left(i \alpha_{2}, i \alpha_{1}, i v_{2}, i v_{1}\right)$, where $i$ is a square root of -1 . Conjugation by $w$ is an outer automorphism of $E_{6}$, it preserves a split maximal torus $T \subset E_{6}$, and it fixes a subgroup isomorphic to $F_{4}$ [Ga01, 2.4 and 3.5]. So $V \oplus V^{*}$ is faithful and irreducible as an $E_{6} \rtimes \mu_{4}$ representation, and also as an $N_{E_{6}}(T) \rtimes \mu_{4}$ representation. Also notice that $w^{2}$ is in 
the centre of $E_{7}$. We will denote the set of $T$-weights of $V \oplus V^{*}$ as $\Lambda$, which consists of all weights in $\hat{T}$ of length $4 / 3$.

\section{Essential Dimension}

Definition 1.1. [PV89, 7] Let $k$ be an algebraically closed field, and let $G$ be an algebraic group acting on an irreducible variety $X$ over $k$. We say that $H \subset G$ is a stabilizer in general position (SGP) if there is a dense open subset $U \subset X$ such that $\operatorname{Stab}_{G}(x)$ is conjugate to $H$ for every $x \in U$.

Lemma 1.2 (Popov). Let $X$ and $Y$ be irreducible $G$-varieties. Assume $H_{1}$ is an SGP for the $G$ actions on $X$, and $H_{2}$ is an $S G P$ for the $H_{1}$ action on $Y$. Then $H_{2}$ is an $S G P$ for the $G$ action on $X \times Y$.

Proof. Assume $\operatorname{Stab}_{G}(x)=g^{-1} H_{1} g$ and $\operatorname{Stab}_{H_{1}}(y)=h^{-1} H_{2} h$. Then $\operatorname{Stab}_{G}(x, y)=$ $\operatorname{Stab}_{\operatorname{Stab}_{G}(x)}(y)=\operatorname{Stab}_{g^{-1} H_{1} g}(y)=g h^{1} H_{2} h g^{-1}$.

There is a series of subgroups as follows.

$$
\{1\} \subset \mathrm{SL}_{2} \subset \mathrm{SL}_{3} \subset G_{2} \subset \operatorname{Spin}_{7} \subset \operatorname{Spin}_{8} \subset F_{4} \subset E_{6} \subset E_{7} .
$$

We will abuse notation slightly and use the above symbols to refer to specific subgroups of these types, as described in the proofs of Propositions 1.3 and 1.4.

Several of the results in the following two propositions may be found in $[$ Bo11, 1.5] for the complex numbers, and the arguments there are analogous to the ones used here. For the rest of this section we will assume the characteristic of $k$ is not 2 or 3 .

Proposition 1.3. Assume that $k=\bar{k}$.

(1) $F_{4}$ is an $S G P$ of the $E_{6}$ action on $V$,

(2) $\mathrm{Spin}_{8}$ is an $S G P$ of the $F_{4}$ action on $V$ (or $\left.V^{*}\right)$,

(3) $\operatorname{Spin}_{8}$ is an $S G P$ of the $E_{6} \rtimes \mu_{4}$ action on $V \oplus V^{*}$,

(4) $\operatorname{Spin}_{8} \times \mu_{6}$ is an $S G P$ of the $E_{6} \rtimes \mu_{4}$ action on $\mathbb{P}\left(V \oplus V^{*}\right)$.

Proof. Recall the $E_{6} \rtimes \mu_{4}$ action on the 54 dimensional faithful representation $V \oplus V^{*}$ described above. As in [Ga01,3.5], each of $V$ and $V^{*}$ come equipped with isomorphic Jordan algebra structures, which include a cubic form called the norm. $E_{6}$ preserves both of those cubic forms, but as $E_{6}$ representations $V$ and $V^{*}$ are not isomorphic. The subgroup of $E_{6}$ which fixes the identity element $e$ in $V$ (equivalently, in $V^{*}$ ), is the automorphism group of the Jordan algebra structure, and is of type $F_{4}$ (see also [SV00, 5.9.4 and Section 7]). The subgroup of $F_{4}$ which fixes a chosen triple $\left(e_{1}, e_{2}, e_{3}\right)$ of pairwise orthogonal primitive idempotents in $V$, is isomorphic to $\operatorname{Spin}_{8}$ [Ja68, p.378].

(1) Two elements with non-zero norms in $V$ are in the same $E_{6}$-orbit iff their norms are equal ([Ja68, Chap. IX.6, exer. 6], or [SV00, Proof of Thm. 7.3.2]).

(2) The subgroup $F_{4} \subset E_{6}$ is the group of automorphisms of the Jordan algebra structure on $V$ (which is the same as that on $V^{*}$ ). By Jacobson [Ja68, p.381], any element can be "diagonalized", in other words, the $F_{4}$-orbit of any element non-trivially intersects the subspace spanned by $e_{1}, e_{2}$, and $e_{3}$. If $y$ is in that subspace and its $e_{i}$ coefficients are all distinct (an open condition), then $\operatorname{Stab}_{F_{4}}(w) \cong \operatorname{Spin}_{8}$.

(3) We have $e \in V$, and choose $y \in V^{*}$ as above, such that the norm of $y$ is not a fourth root of unity. Then $(e, y)$ can be considered to be in general position; by Lemma 1.2 , we see that $\operatorname{Spin}_{8}$ is the SGP of $E_{6}$ on $V \oplus V^{*}$. For any $g \in E_{6}$, and $i=1,2,3$, the assumption on the norm of $y$, together with the fact that $E_{6}$ is norm preserving, tells us that $g w^{i}(e, y) \neq(e, y)$. The result follows. 
(4) For $e$ and $y$ as above, consider the element $[e, y] \in \mathbb{P}\left(V \oplus V^{*}\right)$. Notice that $\operatorname{Stab}_{F_{4}}([e, y]) \subset N_{F_{4}}\left(\operatorname{Stab}_{F_{4}}(e, y)\right)=\operatorname{Spin}_{8} \rtimes S_{3}$. Here the symmetric group $S_{3}$ permutes the diagonal coefficients of $e_{i}$ in the Jordan algebra $V$ (and $V^{*}$ ). Since the $e_{i}$ coefficients of $y$ are distinct, we see $\operatorname{Stab}_{F_{4}}([e, y])=\operatorname{Spin}_{8}$. Also $\operatorname{Stab}_{E_{6}}([e])=F_{4} \times \mu_{3}$ $[\mathrm{Ga} 01,3.4]$ (see also $[\mathrm{Ga09}, 9.12]$ ), so $\operatorname{Stab}_{E_{6}}([e, y])=\operatorname{Spin}_{8} \times \mu_{3}$. Finally, if $g \in E_{6}$ such that $g w$ fixes $[e, y]$, then $y$ would have to be a scalar multiple of $e$, which is not the case. Therefore $\operatorname{Stab}_{E_{6} \rtimes \mu_{4}}([e, y])=\operatorname{Spin}_{8} \times \mu_{6}$, where $\mu_{6}$ is the product of the centres of $E_{6}$ and $E_{7}$.

$\operatorname{Spin}_{8}$ has three inequivalent 8 dimensional irreducible representations. We will choose one and call it the vector representation, and denote it by $\Sigma$. Then the other two, $\mathbb{O}^{+}, \mathbb{O}^{-}$, will be called the half-spin representations. Also, $\mathbb{O}$ will denote the 8 dimensional spin representation of $\operatorname{Spin}_{7}$.

Proposition 1.4. Assume $k=\bar{k}$.

(1) $\mathrm{Spin}_{7}$ is an $S G P$ of the $\mathrm{Spin}_{8}$ action on $\Sigma$,

(2) $G_{2}$ is an $S G P$ of the $\operatorname{Spin}_{7}$ action on $\mathbb{O}$,

(3) $\mathrm{SL}_{3}$ is an $S G P$ of the $G_{2}$ action on $\mathbb{O}$,

(4) $\mathrm{SL}_{2}$ is an $S G P$ of the $\mathrm{SL}_{3}$ action on $\mathbb{O}$,

(5) $\mathrm{SL}_{2}$ acts generically freely on $\mathbb{O}$.

Proof. (1) The stabilizer of a point with non-zero norm in the vector representation is a subgroup of $\operatorname{Spin}_{8}$ isomorphic to $\operatorname{Spin}_{7}$. This is the copy of $\operatorname{Spin}_{7} \subset \operatorname{Spin}_{8}$ that we will use below.

(2) As $\operatorname{Spin}_{7}$ representations, $\mathbb{O}^{+}$and $\mathbb{O}^{-}$are isomorphic to $\mathbb{O}$. The claim follows from [Ig70, Prop. 4]; here $G_{2}$ is the automorphism group of an octonion algebra structure on $\mathbb{O}$.

(3) As $G_{2}$ representations, $\Sigma$ and $\mathbb{O}$ are isomorphic. In the 7-dimensional space of elements orthogonal to the identity (also called pure octonions), $G_{2}$ acts transitively on norm one elements, and the stabilizer of such an element $x_{1}$ is $\mathrm{SL}_{3}$ (see also [KMRT, $\S 36$ Exer. 6]).

(4) $\mathrm{SL}_{3}$ acts transitively on norm one elements orthogonal to both the identity and $x_{1}$. The stabilizer of such an element $x_{2}$ is $\mathrm{SL}_{2}$ (see also [KMRT, §36 Exer. 7]).

(5) $\mathrm{SL}_{2}$ acts transitively on norm one elements orthogonal to all of $1, x_{1}, x_{2}$, and $x_{1} x_{2}$. The stabilizer of such an element $x_{3}$ is trivial, because an automorphism of the octonions is determined by its action on the "basic triple" $\left(x_{1}, x_{2}, x_{3}\right)$, and therefore $\mathrm{SL}_{2}$ acts generically freely on $\mathbb{O}$.

Remark 1.5. As noted in [Ig70, Prop. 1], if $H$ is an SGP of $\operatorname{Spin}_{8}$ acting on the half-spin representation $\mathbb{O}^{+}$, then $H$ is isomorphic (but not conjugate) to $\operatorname{Spin}_{7}$ by a triality automorphism. And the $\operatorname{Spin}_{8}$ representation $\Sigma$ restricted to $H$ is the spin representation (rather than the vector $\mathrm{Spin}_{7}$-representation).

Theorem 1.6. $\operatorname{ed}\left(E_{7}\right) \leq 29$.

Proof. From the introduction, we know that $\operatorname{ed}\left(E_{7}\right) \leq \operatorname{ed}\left(E_{6} \rtimes \mu_{4}\right)$. Consider the rational map

$$
\left(V \oplus V^{*}\right)^{2} \rightarrow W:=V \oplus V^{*} \times \mathbb{P}\left(V \oplus V^{*}\right) .
$$

It is dominant and $E_{6} \rtimes \mu_{4}$-equivariant. To show the upper bound on essential dimension, we just need to show that $W$ is generically free, and for this we may assume that $k=\bar{k}$.

By Lemma 1.2 and Proposition 1.3, we see that an SGP of the $E_{6} \rtimes \mu_{4}$ action on $W$ is equal to an SGP of the $\operatorname{Spin}_{8}$ action on $V \oplus V^{*}$. As a $\operatorname{Spin}_{8}$ representation this 
decomposes as the sum of six trivial representations, together with two copies each of $\Sigma, \mathbb{O}^{+}$, and $\mathbb{O}^{-}$. Therefore, by combining Lemma 1.2 with Proposition 1.4 , we see the SGP of this $\operatorname{Spin}_{8}$ action is trivial, and hence the action of $E_{6} \rtimes \mu_{4}$ on $W$ is generically free. Therefore ed $\left(E_{7}\right) \leq \operatorname{ed}\left(E_{6} \rtimes \mu_{4}\right) \leq 54+53-78=29$.

\section{Essential 2-DiMENSION}

Lemma 2.1. [MR09, Lemma 3.3] Assume we have algebraic groups $1 \rightarrow T \rightarrow N \rightarrow$ $F \rightarrow 1$, such that $T$ is a split torus, and $F$ is finite. Let $V$ be a faithful $N$-representation which decomposes into a sum of one-dimensional weight spaces for $T$, and let $\Lambda$ be the associated set of weights. Consider the $N$-invariant open subset $U=\left(\mathbb{A}^{1}-0\right)^{\Lambda} \subset \mathbb{A}^{\Lambda}=$ $V$.

(1) If $f \in F$ acts non-trivially on $\operatorname{ker}(\mathbb{Z}[\Lambda] \rightarrow \hat{T})$, then $f$ acts non-trivially $U / T$.

(2) $N / T$ acts faithfully on $U / T$ if and only if $N$ acts generically freely on $V$.

Proof. For the first statement, assume $f$ acts trivially on $U / T$ and choose an arbitrary $\Sigma c_{\lambda} \lambda \in \operatorname{ker}(\mathbb{Z}[\Lambda] \rightarrow \hat{T})$. Choose an isomorphism between the varieties $U \cong\left(\mathbb{G}_{m}\right)^{\Lambda}$, inducing a multiplicative structure on $U$ (which is not necessarily compatible with the action of $N$ ). Consider the functions $U \rightarrow k^{*}$ defined in terms of the $\lambda$-coordinates $u_{\lambda}$ of an arbitrary $u \in U$, as

$$
\phi_{1}(u)=\prod_{\lambda \in \Lambda}\left(u_{\lambda}\right)^{c_{\lambda}}, \quad \phi_{2}(u)=\prod_{\lambda \in \Lambda}\left(u_{f \cdot \lambda}\right)^{c_{\lambda}} .
$$

These functions are invariant under the action of $T$ on $U$. Now choose $n_{f} \in N$ in the preimage of $f$. Since $n_{f}$ acts linearly on $V$, there are constants $b_{\lambda}$ and $c$ such that $\phi_{1}(u)=\phi_{1}\left(n_{f} \cdot u\right)=\prod\left(b_{\lambda} u_{f \cdot \lambda}\right)^{c_{\lambda}}=c \phi_{2}(u)$. Since $u \in\left(\mathbb{G}_{m}\right)^{\Lambda}$ was arbitrary, the only way $\phi_{1}$ and $\phi_{2}$ can be constant multiples of each other is if $c_{\lambda}=f \cdot c_{\lambda}$ for every $\lambda \in \Lambda$; in other words, $f$ fixes $\Sigma c_{\lambda} \lambda$.

For the second statement, assume $N / T$ acts faithfully on $U / T$. For each non-trivial $f \in N / T$, choose a representative $n_{f} \in N$. The action of $f$ on $U / T$ is non-trivial, and therefore there is an open dense set $U_{f} \subset U$ on which $n_{f} t$ has no fixed points for any $t \in T$. The finite intersection $\cap U_{f}$ is open and dense in $U$, and $N$ acts with trivial stabilizers on it. In other words, $N$ acts generically freely on $U$, and hence on $V$. For the converse, we just need to notice that a faithful representation of a finite group is always generically free.

Remark 2.2. The above Lemma was proved in [MR09] when the exact sequence is split, in which case the converse of the first statement is also true; the standard representation of $N_{\mathrm{SL}_{2}}(T)$ is a counter-example to the converse of the first statement.

Theorem 2.3. $\operatorname{ed}\left(E_{7} ; 2\right) \leq 27$.

Proof. This upper bound will be proved using the following sequence of inequalities:

$$
\operatorname{ed}\left(E_{7} ; 2\right) \leq \operatorname{ed}\left(E_{6} \rtimes \mu_{4} ; 2\right) \leq \operatorname{ed}\left(N_{E_{6}}(T) \rtimes \mu_{4} ; 2\right) \leq 33-6=27 .
$$

Since conjugation by $\mu_{4}$ preserves $T([\mathrm{Ga} 01,2.4])$, we have $N_{E_{6} \rtimes \mu_{4}}(T)=N_{E_{6}}(T) \rtimes \mu_{4}$. Now the middle inequality follows from [Se02, III.4.3, Lemma 6].

Choose a Sylow 2-subgroup $W^{(2)} \subset W\left(E_{6}\right)$, and let $N_{2} \subset N_{E_{6}}(T) \rtimes \mu_{4}$ be the preimage of $F:=W^{(2)} \rtimes \mu_{4}$. Let $\sigma$ be the square of a generator of $\mu_{4}$; it is the nontrivial element in the centre of $E_{7}$. One can verify the following facts using a computer algebra program such as Magma.

- The $F$ orbits of $\Lambda$ are of size $2,4,16$ and 32 .

- The weights in the largest orbit $\Lambda_{32}$ additively generate the weight lattice 
- The kernel of the $F$ action on $\operatorname{ker}\left(\mathbb{Z}\left[\Lambda_{32}\right] \rightarrow \hat{T}\right)$ is $\langle\sigma\rangle$.

So the subspace $V_{32} \subset V \oplus V^{*}$ generated by the weight spaces of $\Lambda_{32}$ is an irreducible $N_{2}$-representation of dimension 32 . Let $V_{1}$ be the one-dimensional non-trivial representation of $\mu_{4}$, which we also consider as an $N_{2}$ representation via projection to $\mu_{4}$. We claim that $N_{2}$ acts generically freely on $V_{32} \times V_{1}$.

For $U \subset V_{32} \times V_{1}$ as in the above Lemma, the third point implies that the $F$ action on $U / T$ has kernel contained in $\langle\sigma\rangle$. But $\sigma$ acts non-trivially on $V_{1}$, and hence on $U / T$, since $T$ acts trivially on $V_{1}$. So $F$ acts faithfully on $U / T$, and hence by Lemma 2.1, the $N_{2}$ action on $V_{32} \times V_{1}$ is generically free. Now we have the upper bound $\operatorname{ed}\left(N_{E_{6}}(T) \rtimes \mu_{4} ; 2\right) \leq 33-6=27$, and the result follows.

\section{REFERENCES}

[BF03] G. Berhuy, G. Favi, Essential dimension: a functorial point of view (after A. Merkurjev). Doc. Math. 8 (2003), 279-330.

[Bo11] C. Boehning, The rationality problem in invariant theory, preprint online.

[Bo92] A. Borel, Linear algebraic groups. Second edition. Graduate Texts in Mathematics, 126. Springer-Verlag, New York, (1991).

[BR97] J. Buhler, Z. Reichstein, On the essential dimension of a finite group. Compositio Math. 106 (1997), no. 2, 159-179.

[CS06] V. Chernousov, J.-P. Serre, Lower bounds for essential dimension via orthogonal representations. J. Algebra 305 (2006), 1055-1070.

[Ga01] S. Garibaldi, The Rost invariant has trivial kernel for quasi-split groups of low rank, Comment. Math. Helv. 76 (2001) 684-711.

[Ga09] S. Garibaldi, Cohomological invariants: Exceptional groups and Spin groups, Memoirs of the American Mathematical Society, Number 937 (2009).

[GR09] P. Gille, Z. Reichstein, A lower bound on the essential dimension of a connected linear group, Comment. Math. Helv. 84, No. 1 (2009) 189-212.

[Ig70] J. Igusa, A classification of spinors up to dimension twelve, Amer. J. Math. 92 (1970), 997-1028.

[Ja68] N. Jacobson, Structure and representations of Jordan algebras. Amer. Math. Soc. Colloq. Publ. vo. XXXIX (1968).

[KMRT] M.-A. Knus, A. Merkurjev, M. Rost, J.-P. Tignol, The book of involutions, Colloquium Publications Volume 44, American Mathematical Society (1998).

[Le04] N. Lemire, Essential dimension of algebraic groups and integral representations of Weyl groups, Transform. Groups 9 (2004), no. 4, 337-379.

[Mac11] M.L. MacDonald, Essential p-dimension of the normalizer of a maximal torus, Transform. Groups 16, (2011), no. 4, 1143-1171

[MR09] A. Meyer, Z. Reichstein, Essential dimension of the normalizer of an maximal torus in the projective linear group, Algebra \& Number Theory 3 (2009), no. 4, 467-487.

[Po86] A.M. Popov, Finite isotropy subgroups in general position of simple linear Lie groups, Trans. Mosov Math. Soc. (1986), 3-63.

[PV89] V.L. Popov, E.B. Vinberg, Invariant Theory. Algebraic Geometry IV, Encyclopaedia of Mathematical Sciences 55, Springer, (1989). Translated from the Russian.

[Re10] Z. Reichstein, Essential dimension, ICM proceedings, (2010).

[RY00] Z. Reichstein, B. Youssin, Essential dimensions of algebraic groups and a resolution theorem for $G$-varieties. With an appendix by János Kollár and Endre Szabó. Canad. J. Math. 52 (2000), no. 5, 1018-1056.

[Se02] J.-P. Serre. Galois cohomology. Translated from the French by Patrick Ion and revised by the author. Corrected reprint of the 1997 English edition. Springer Monographs in Mathematics. Springer-Verlag, Berlin, (2002).

[SV00] T.A. Springer, F.D. Veldkamp, Octonions, Jordan algebras and exceptional groups. Springer (2000). 